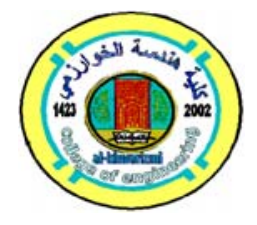

Al-Khwarizmi Engineering Journal, Vol. 17, No. 1, March, (2021)

P. P. 34- 42

\title{
Hurst Exponent and Tsallis Entropy Markers for Epileptic Detection from Children
}

\author{
Sumai Hamad Jaafer \\ Erbil Medical Institute/ Erbil Polytechnic \\ Email: sumaya.hamad@epu.edu.iq
}

(Received 2 August 2020; accepted 23 November 2020)

https://doi.org/10.22153/kej.2021.11.001

\begin{abstract}
The aim of the present study was to distinguish between healthy children and those with epilepsy by electroencephalography (EEG). Two biomarkers including Hurst exponents $(\mathrm{H})$ and Tsallis entropy (TE) were used to investigate the background activity of EEG of 10 healthy children and 10 with epilepsy. EEG artifacts were removed using Savitzky-Golay (SG) filter. As it hypothesize, there was a significant changes in irregularity and complexity in epileptic EEG in comparison with healthy control subjects using t-test $(\mathrm{p}<0.05)$. The increasing in complexity changes were observed in $\mathrm{H}$ and TE results of epileptic subjects make them suggested EEG biomarker associated with epilepsy and a reliable tool for detection and identification of this disease in children. Generally, this study aims to provide an evident of a reliable biomarker to detect epilepsy in children to give them an additional chance to live a high quality life.
\end{abstract}

Keywords: Detection, Electroencephalography, Entropy, Epilepsy, Savitzky-Golay, t-test.

\section{Introduction}

Epilepsy is a very common and old neurological disease, with around 50 million patients around the world $80 \%$ of them in the developing countries [1]. The clinical detection of the epilepsy depends on the history of the family beside a thoroughly analysis of the EEG test for abnormal changes. Epilepsy, according to the International League Against Epilepsy (ILAE), is a brain disorder in which there is continuous propensity to start an epileptic seizures and by the cognitive, neurobiological, social, and psychological consequences of this condition.

It is required to have one epileptic seizure to consider the case as epileptic subject, this seizure leads to a temporal disorders and symptoms occurs on the brain due to excessive, abnormal, and hypersynchronous neural discharge activity in the brain, which can cause brain dysfunctional, the word came from Greek meaning to take hold [2]. Patients with epilepsy may suffer from cognitive problems such as stigma, exclusion, restrictions, overprotection, and isolation, which also become part of the epileptic condition. Seizures sometimes lead to unexpected death during or after a period of ictus, normally these types of deaths categorized as sudden death, regarding a known clinical cause of death [3], most probably due to asphyxia [4].

Epilepsy can originate in different locations and lobes in the brain, normally all located in the brain cortex, for example in $[5,6]$ the seizures originated in the frontal lobe, most of the mentioned cases where originated from partial seizures., The studied cases included occurrence of seizures while sleeping, mainly during the rapid eye movement (REM) stage. In [7], a case study of a 18 months old infant, has a seizures in his cerebellar ganglioma, due to the existence of a mass in his left cerebellar hemisphere, which leads to a hemifacial contractions in his left side of his face. Temporal Lobe Epilepsy (TLE) is also 
a recorded cases and had been studied over women on reproductive intervals [8].

Epilepsy is a significant neurological disorder that is identified by recurrent spontaneous partial or generalized seizures. It is estimated that the proportion of the global population with active epilepsy is between 4 and 10 per 1000 people. This proportion is getting higher and higher in developing countries and can reach 6 to 10 per 1000 people. Epileptic seizures vary from shortterm twitches to prolonged and severe convulsions they could happen at any age as well as at different levels of severity. Even though there is progress in the types of available epilepsy treatment, about $30-40 \%$ of epilepsy patients fail to respond to antiepileptic drugs or other types of interventions. Taking into consideration that even patients who respond to the available epilepsy treatments may encounter complications and medical issues such as depression, it is important to understand the complex mechanisms and factors that may influence on epilepsy. This understanding could lead to novel treatments that would modify its generation/progression and eventually control or reduce seizures in epilepsy patients, thus avoiding at least some of the medical complications associated with current available treatment.

Electroencephalogram (EEG) is a very stochastic, non-stationary and highly complex signal that has been widely used to detect epilepsy. EEG signals with such a nature makes it difficult to be analyzed using normal clinical inspection or time domain techniques [9]. Automated detection of epilepsy is done by making a clear distinguishing between ictal and interictal intervals of an epileptic EEG signal, in this manner a reliable epileptic detector will be available, which can perform an online detection of the recorded EEG signal with a perfect diagnosis for the patient.

Normally the detection of epileptic patients done by clinical inspection and analysis of the EEG recording of each patient, yet this process consumes a lot of time and effort while the needs to shorten the time required due to the increment in the number of patients [10]. Another important issue is the accuracy of the clinical test, which require the presence of a professional each time, otherwise will effect on the quality of diagnosis, while the automated detection will ensure a high accuracy and reliable diagnosis each time and in all conditions [11].

The traditional way to detect epileptic spikes is by using EEG recordings through the visual scanning and inspection. However, this technique required long EEG diagnostic time and it is an inaccurate method. To overcome these cons, many automatic methods have been used such as linear time-frequency analysis and non-linear such as largest Laypunov exponent (LLE) and correlation dimension (CD) [12, 13]. Furthermore, several types of EEG measurements have been used due to complexity of the brain and the signal such as approximate entropy (AE) [14], Kolmogorov entropy [15], sample entropy [16, 17]. In addition, many available system today such as long term epilepsy, cEEG Intensive care unit (ICU) monitoring and sleep testing for the most flexible and comprehensive recording system and long legacy.

In the current study, the Hurst exponents $(\mathrm{H})$ and Tsallis entropy (TE) have been computed to enhance the epileptic process understanding and to distinguish the children with epilepsy from the control healthy subjects. The t-test has been used to show the reliability of the new methods to measure the complexity-based structure of the brain for children with epilepsy and healthy ones and to find the significant differences among various groups at $\mathrm{p}<0.05$ significance level.

\section{Materials and Methods}

Figure 1 shows the block diagram of the proposed study.

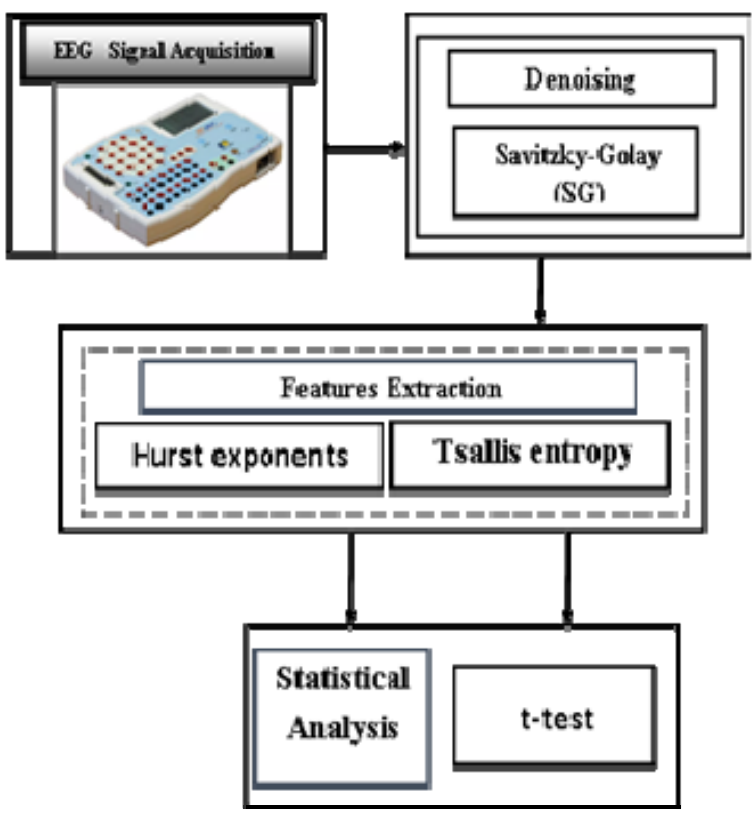

Fig. 1. The block diagram of the current study. 


\subsection{Procedure of Recording and Acquisition of EEG Data}

EEG data has been obtained by using EEG recording headset (a Micromed Brain Spy Plus Embia via Giotto 2, 31021 Mogliano Veneto, Treviso, Italy). Two reference electrodes, with 19 electrodes in addition to one ground electrode were used. Notably, international system of 10-20 has been applied, thus following arrangement of the EEG electrodes arrangement was performed (FP1, FP2, F7, F3, Fz,F4, F8, T3, C3, Cz, C4, T5, $\mathrm{P} 3, \mathrm{Pz}, \mathrm{P} 4, \mathrm{~T} 6, \mathrm{O} 1$ and $\mathrm{O} 2$ ). Importantly, electrode reluctance has been fixed to be below 5 kilo-Ohms. EEG dataset of the current study have been obtained by analyzing samples of 20 children, who they are primary school students (10 normal control students, and 10 students with epilepsy). Students' ages were from 6 to 12 years old. Controls students had not shown any conditions such as psychiatric or neurological. Notably, human ethics committee of the teaching hospital, Erbil, Iraq has approved all protocols were applied in this experiment. Children who are suffering from epilepsy have been chosen from the Teaching Hospital Neurology Clinic. Finally, all participants in this study signed an information consent form (ICF).

\subsection{Denoising Stage}

Previous studies have demonstrated that noise from both extrinsic and intrinsic sources, for example noise resulted from surrounding environment, and noise producing by internal structure of human body have to be eradicated [18-20]. Therefore, removing power line interference noise has been performed by using notch filter. Consistently, butter worth has been utilized for the second order band pass filter ranging from 0.1 to $60 \mathrm{~Hz}$ in the first stage [21]. In the second stage, and in order to smooth out the signal with lowest level of destruction of its original features, Savitzky-Golay (SG) has been utilized. Interestingly, shifting effect after filtering the signal has been involved in this approach. One of the important features of SG filter, it offers advantages of preserving features of a time series that includes its relative minima and maxima, which indicate a highly significant concern relating to segmentation of a signal such as EEG [22-25].

It has been reported that in two parameters, the performance of SG filter mainly dependents on the frame size and polynomial order. Importantly, following equation has been depended on to fit the coefficients of an SG filter $(\mathrm{N}=\mathrm{Nr}+\mathrm{N} 1+1)$ points of the EEG signal. So window size described by $\mathrm{N}$, while both signal points in right and signal points in left of a current signal point have been identified by $\mathrm{Nr}$ and $\mathrm{N} 1$ respectively. Moreover, it has been reported that set up investigation of SG filter parameters to the 3 rd order polynomial and a frame size of 51 samples is essential to de-noise and smooth the EEG dataset [26].

\subsection{Features Extraction}

Epilepsy is a central nervous system disorder that is accompanied by frequent seizures. EEG is the main recording method that has been used for a long time for seizures events recoding. However, EEG is not very efficient for diagnosing, and classification of different conditions such as normal, predictive, and psychological conditions, which are relatively difficult due to the instability of EEG signals and the length of the recording period. This deficiency of the previously mentioned method required the researchers to try new techniques. In this study, two techniques these are Hurst Exponents $(\mathrm{H})$ and Tsallis Entropy (TE) were suggested for the detection of epileptic seizures.

$\mathrm{H}$ was used by Kannathal et al. as a chaotic measure, among other techniques, as a measure of signal prediction. The percentage accuracy of these measurements about $90 \%$ in detecting epileptic EEG This test is an important indicator of long-term signal recording. The value of the Hearst Exponent is specified between 0 and 1, and the next deviation in the sign is determined by this value [27]. In other words, the self-similarity and correlation of a signal can be assessed by $\mathrm{H}$. This parameter could also reveal the fractal time series flatness depending on the asymptotic behavior of the rescaled variety of the procedure. The calculation method as follows:

$$
\mathrm{H}=\frac{\log \left(\frac{R}{S}\right)}{\log (T)}
$$

where $\mathrm{R} / \mathrm{S}$ represents corresponding rescaled range value, $\mathrm{R}$ the deviation, minimum and maximum, away from the mean. $\mathrm{S}$ is the standard deviation, and $\mathrm{T}$ is the sample data [26].

In general, earlier, the entropy is defined as a measure based on information theory to calculate order and disorder in a dynamic system [27-28]. This technique could be preferable in monitoring 
signals disorder because the injured brain has a low entropy compared to random continuous EEG.

TE considers as a nonextensive statistical mechanics [27]. This technique is perfect as a tool to define long-term memory effects systems, multifractal space-time constraints, or long-range interactions [28]. TE has its scaling system could utilize to investigate short- and long-range interactions. Thus, the Tsallis statistics help differentiate among a range of EEG outcomes such as spikes, bursts, and continuous or fused rhythms. The equation that calculates the value of TE is:

$$
\text { TsEn }=\frac{1-\sum_{i=1}^{W} \quad p_{i}^{q}}{q-1}
$$

where q represents the nonextensivity degree.

The TE was firstly presented to the generalization of nonextensivity in the thermodynamical sense. However, recently, it gets broader applications, and it is widely applied in biomedical signal processing such ECG analysis and, most recently, EEG .The resent studies postulate that the Tsallis statistics deliver additional detailed info compared to the conventional procedures, particularly in spike or burst EEG analysis [28]. Recent work shows the accuracy of Tsallis Entropy of 92.67$100 \%$ in detecting different patterns of EEG signals in various types of problems. Furthermore, the problem-solving time for this method is $\sim 0.9$ $\mathrm{ms}$, i.e., real-time detection.

In order to distinguish between epileptic EEG signals and normal healthy control subjects, two types of entropy have been computed in this study. These types are these are TE and H. In order to extract features from the original EEG time series for each 19 channels, 60 seconds, $\mathrm{N}=15360$ samples, concomitant with 6 windows of 10 second length (2560 samples) were used in the current study. Furthermore, EEGs' abnormalities of dementia patients have been detected by using Entropies.

Previous researchers have revealed that TsEn is non-extensive, it was first introduced by Havrda and Charvát then examined in details by Daróczy $[28,29]$. Actually, measurement of this entropy completely differs from that measurement by standard Boltzman-Gibbs-Shannon entropy measure. In the same line of thought, the Boltzman-Gibbs-Shannon measure has been widespread to a group of measures that are not necessarily comprehensive. Alternatively, they can be either sub-additive or super-additive.
Furthermore, TsEn with parameter have been utilized. Thus, a generalized measurement of entropy, due to TsEn, can be assessed as mentioned by both [30-31]. The concept is based on the measure of the relative frequencies of different motifs. It has been revealed that Hurst Exponent $(\mathrm{H})$ could be majorly used to calculate the self-similarity of the time series like EEG [17][32].

\subsection{Statistical analysis}

Considering the scalp area of the cerebral cortex, denoised 19 channels from the EEG dataset of ten control (healthy), and ten patient's children with epilepsy have grouped into been categorized into five groups. Consequently, these regions represented frontal channels (seven) (Fp1, Fp2, F3, F4, F7, F8, and Fz), three parietal channels (P3, P4, and Pz), both temporal channels (T3 and T5), two occipital channels (O1 and $\mathrm{O} 2$ ), and three central channels $(\mathrm{C} 3, \mathrm{C} 4$, and $\mathrm{Cz})$. Thereafter, Kolmogorov-Smirnov test was applied in order to measure normality. On the other hand, Levene's test was used to prove homoscedasticity. Therefore, t-test has been performed as a statistical way using SPSS 23. In all sections the independent variables (IV) were the subject groups (control healthy normal subjects and children with epilepsy) as the first IV and the five scalp regions (frontal, temporal, parietal, occipital and central) as the second IV. Whilst, one of the former features was the dependent variable (DV). Notably, significance for all statistical tests was set at $\mathrm{p}<0.05$.

\section{Results and Discussion}

EEG data were recorded for the healthy control subjects and epileptic children as shown in (Figures 2 and 3), respectively. Form the visual inspection of the waveforms, the recorded EEG signals were exhibits patterns of normal or abnormal brain electrical activities. Abnormal patterns may occur because of seizure effect on the brain. 


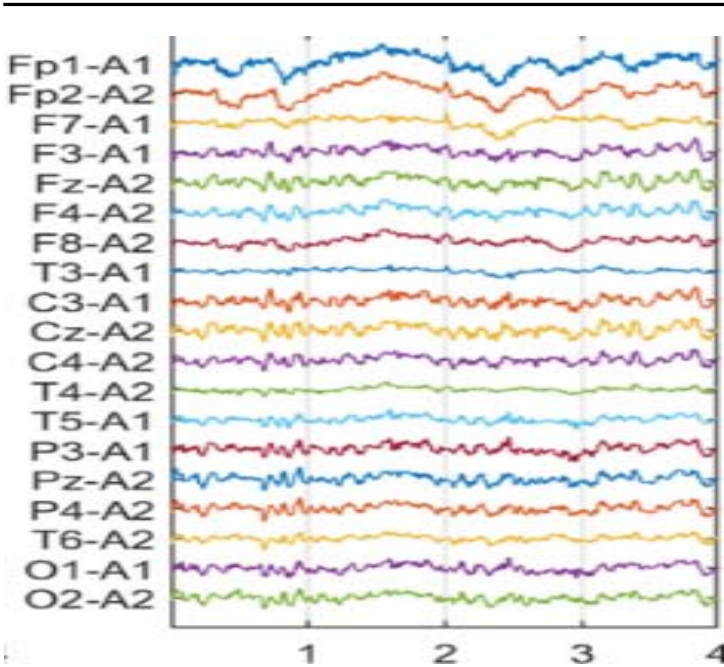

Fig. 2. The EEG activities for the first control healthy subject.

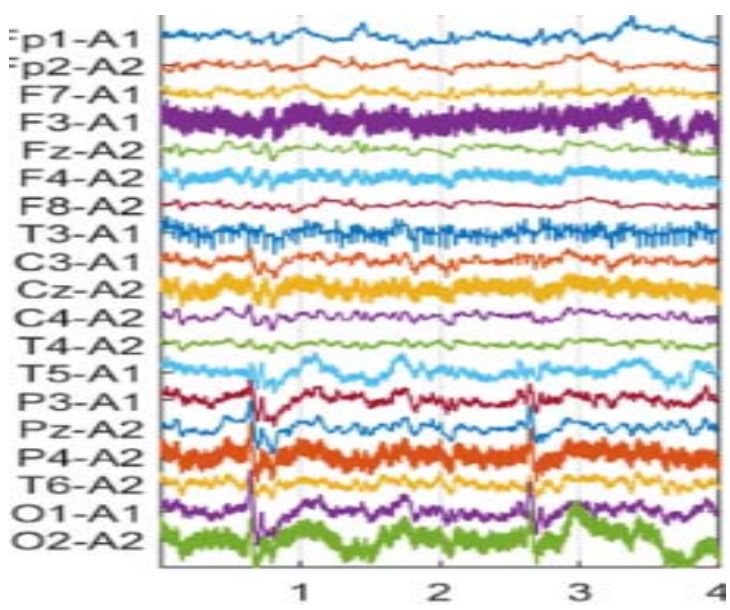

Fig. 3. The EEG activities for the first subject with epilepsy.

The observation to Figure 4 shows that SG filters were smoothed the recorded EEG dataset. There were suppressing for all components of the noise as shown in channel $2(\mathrm{Ch} 2)$, blue colored line, compared with the original noisy recorded EEG in red colored line.

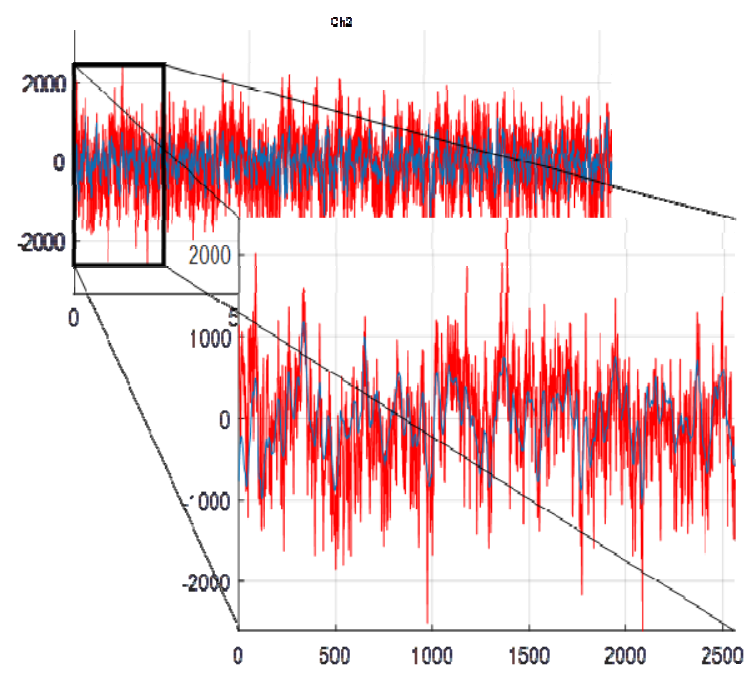

Fig. 4. After using SG filter for $\mathrm{Ch} 2$ which represent Fp2 to denoising.

Table 1 demonstrates the $\mathrm{H}$ and TE entropy values for the children with epilepsy and agematch control subjects over the five scalp regions. It can be noted that the $\mathrm{H}$ of epileptic children EEG signals is slightly higher than that of normal EEG signals in frontal, temporal and occipital regions. This might resulted from hyper mental activity for epileptic children compare to those with normal brain. As well as due to the high regularity and complexity of the EEGs during the epileptic seizure compared to normal EEGs. On the other hand, the value of TE indicated high enactment in order to distinguish normal subjects from epileptic subjects in comparison to $H$. interpretation of this might to some extent relative the EEG recording dynamic processes, which much less complex for people with epileptic than for normal ones. Interestingly, these observations were in agreement with aforementioned works that EEG complexity would be declined as a result of neurotransmitter deficiency, neuronal death and/or loss of connectivity due to death of nerve cell. Notably, recognition between healthy and epileptic subject's EEG signals have a vital role in relative to clinical relevance. Mainly because it provides cooperative outfits for the detection of a seizure onset. Hence, the $\mathrm{H}$ and TE can benefit understand epileptic children and normal ones EEGs. 
Table 1,

The average values (Mean \pm SD) of EEGs for the normal control subjects and epileptic patients over the brain regions. Significant group differences are marked with an asterisk.

\begin{tabular}{llllll}
\hline Brain lobes & Subjects & TE & p-value & H & p-value \\
\hline \multirow{2}{*}{ Frontal } & Normal & $10.73 \pm 0.47$ & $0.05^{*}$ & $0.85 \pm 0.04$ & $0.03^{*}$ \\
& Abnormal & $10.18 \pm 1.19$ & $0.05^{*}$ & $0.86 \pm 0.07$ & $0.03^{*}$ \\
\multirow{2}{*}{ Temporal } & Normal & $10.77 \pm 0.36$ & $0.05^{*}$ & $0.85 \pm 0.03$ & $0.03^{*}$ \\
& Abnormal & $10.22 \pm 1.09$ & $0.05^{*}$ & $0.86 \pm 0.07$ & $0.03^{*}$ \\
Central & Normal & $10.75 \pm 0.37$ & $0.05^{*}$ & $0.85 \pm 0.03$ & 0.06 \\
& Abnormal & $10.21 \pm 1.17$ & $0.05^{*}$ & $0.86 \pm 0.06$ & 0.06 \\
Parietal & Normal & $10.77 \pm 0.34$ & $0.05^{*}$ & $0.85 \pm 0.03$ & 0.07 \\
& Abnormal & $10.18 \pm 1.19$ & $0.05^{*}$ & $0.86 \pm 0.07$ & 0.07 \\
Occipital & Normal & $10.73 \pm 0.4$ & $0.05^{*}$ & $0.85 \pm 0.03$ & $0.03^{*}$ \\
& Abnormal & $10.18 \pm 1.1$ & $0.05^{*}$ & $0.87 \pm 0.06$ & $0.03^{*}$ \\
\hline
\end{tabular}

\section{Conclusions}

In summary, this work was attempted to discernment between children with epilepsy and healthy normal subjects. SG filter has been employed for denoising and smoothing the EEG dataset; and $\mathrm{H}$ and TE were computed. Statistical analysis using t-test has been conducted to characterize the features and to investigate the presented hypothesis that both irregularity and complexity in epileptic EEG. In fact, reducing the complexity in epileptic patients compare to the healthy control mainly derived by TE whereas increasing the complexity in epileptic patients compare to the healthy control mainly derived by $\mathrm{H}$. Thus, it can be considered that the $\mathrm{H}$ and TE as essential EEG biomarkers in order to detect and identify irregular seizures for children patients with epilepsy. Importantly, EEG could be used as an important indicator for examining the background activity in the documentation of children patients with epilepsy.

\section{Acknowledgments}

The author wish to express their gratitude to Neurology Unit staff from the Neurology Unit at teaching hospital, Erbil, Iraq for their assistance in the Collecting the EEG brain signals during working for manuscript and provided the neuropsychological assessment for all subjects.

\section{References}

[1] H. M. De Boer, M. Mula, and J. W. Sander, "The global burden and stigma of epilepsy," Epilepsy \& behavior, vol. 12, pp. 540-546, 2008.
[2] E. Trinka, H. Cock, D. Hesdorffer, A. O. Rossetti, I. E. Scheffer, S. Shinnar, S. Shorvon, and D. H. Lowenstein, "A definition and classification of status epilepticus-Report of the ILAE Task Force on Classification of Status Epilepticus," Epilepsia, vol. 56, pp. 1515-1523, 2015.

[3] D. Baker, "Psychological perspectives on stigma and self-compassion in adults with epilepsy," Lancaster University, 2017.

[4] C. Carrick, K. A. Collins, C. J. Lee, J. A. Prahlow, and J. J. Barnard, "Sudden death due to asphyxia by esophageal polyp: two case reports and review of asphyxial deaths," The American journal of forensic medicine and pathology, vol. 26, pp. 275-281, 2005.

[5] P. Ryvlin, L. Minotti, G. Demarquay, E. Hirsch, A. Arzimanoglou, D. Hoffman, M. Guénot, F. Picard, S. Rheims, and P. Kahane, "Nocturnal hypermotor seizures, suggesting frontal lobe epilepsy, can originate in the insula," Epilepsia, vol. 47, pp. 755-765, 2006.

[6] P. Williamson, V. Thadani, T. Darcey, D. Spencer, S. Spencer, and R. Mattson, "Occipital lobe epilepsy: clinical characteristics, seizure spread patterns, and results of surgery," Annals of Neurology: Official Journal of the American Neurological Association and the Child Neurology Society, vol. 31, pp. 3-13, 1992.

[7] U. Löbel, D. Ellison, B. Shulkin, and Z. Patay, "Infiltrative cerebellar ganglioglioma: conventional and advanced MRI, proton MR spectroscopic, and FDG PET findings in an 18-month-old child," Clinical radiology, vol. 66, pp. 194-201, 2011.

[8] V. V. Kalinin and E. V. Zheleznova, "Chronology and evolution of temporal lobe epilepsy and endocrine reproductive dysfunction in women: relationships to side of 
focus and catameniality," Epilepsy \& Behavior, vol. 11, pp. 185-191, 2007.

[9] N. K. Al-Qazzaz, S. H. B. Ali, S. A. Ahmad, K. Chellappan, M. S. Islam, and J. Escudero, "Role of EEG as Biomarker in the Early Detection and Classification of Dementia," The Scientific World Journal, vol. 2014, 2014.

[10] L. M. Patnaik and O. K. Manyam, "Epileptic EEG detection using neural networks and post-classification," Computer methods and programs in biomedicine, vol. 91, pp. 100109, 2008.

[11] S. Ramgopal, S. Thome-Souza, M. Jackson, N. E. Kadish, I. S. Fernández, J. Klehm, W. Bosl, C. Reinsberger, S. Schachter, and T. Loddenkemper, "Seizure detection, seizure prediction, and closed-loop warning systems in epilepsy," Epilepsy \& behavior, vol. 37, pp. 291-307, 2014.

[12] A. Mirzaei, A. Ayatollahi, and A. M. Nasrabadi, "Automated detection of epileptic seizures using mixed-methodology: WaveletChaos-KNN Classifier-Mutual Information," Electr. Rev, vol. 87, p. 220À23, 2011.

[13] I. I. Andreadis, G. A. Giannakakis, C. Papageorgiou, and K. S. Nikita, "Detecting complexity abnormalities in dyslexia measuring approximate entropy of electroencephalographic signals," in 2009 Annual International Conference of the IEEE Engineering in Medicine and Biology Society, 2009, pp. 6292-6295.

[14] N. K. Al-Qazzaz, S. Ali, S. A. Ahmad, and J. Escudero, "Stroke-related mild cognitive impairment detection during working memory tasks using EEG signal processing," in 2017 Fourth International Conference on Advances in Biomedical Engineering (ICABME), 2017, pp. 1-4.

[15] F. C. Morabito, D. Labate, F. La Foresta, A. Bramanti, G. Morabito, and I. Palamara, "Multivariate multi-scale permutation entropy for complexity analysis of Alzheimer's disease EEG," Entropy, vol. 14, pp. 11861202, 2012.

[16] S.-D. Wu, P.-H. Wu, C.-W. Wu, J.-J. Ding, and C.-C. Wang, "Bearing fault diagnosis based on multiscale permutation entropy and support vector machine," Entropy, vol. 14, pp. 1343-1356, 2012.

[17] N. K. Al-Qazzaz, S. Ali, M. S. Islam, S. A. Ahmad, and J. Escudero, "EEG markers for early detection and characterization of vascular dementia during working memory tasks," in 2016 IEEE EMBS Conference on
Biomedical Engineering and Sciences (IECBES), 2016, pp. 347-351.

[18] N. K. Al-Qazzaz, S. H. M. Ali, and S. A. Ahmad, "Comparison of the Effectiveness of AICA-WT Technique in Discriminating Vascular Dementia EEGs," in 2018 2nd International Conference on BioSignal Analysis, Processing and Systems (ICBAPS), 2018, pp. 109-112.

[19] N. K. Al-Qazzaz, S. Hamid Bin Mohd Ali, S. A. Ahmad, M. S. Islam, and J. Escudero, "Automatic Artifact Removal in EEG of Normal and Demented Individuals Using ICA-WT during Working Memory Tasks," Sensors, vol. 17, p. 1326, 2017.

[20] N. K. Al-Qazzaz, S. H. B. M. Ali, S. A. Ahmad, M. S. Islam, and J. Escudero, "Discrimination of stroke-related mild cognitive impairment and vascular dementia using EEG signal analysis," Medical \& Biological Engineering \& Computing, pp. 121, 2017.

[21] N. K. Al-Qazzaz, M. K. Sabir, S. H. B. M. Ali, S. A. Ahmad, and K. Grammer, "Electroencephalogram Profiles for Emotion Identification over the Brain Regions Using Spectral, Entropy and Temporal Biomarkers," Sensors, vol. 20, p. 59, 2020.

[22] H. Azami, M. Rostaghi, D. Abásolo, and J. Escudero, "Refined composite multiscale dispersion entropy and its application to biomedical signals," IEEE Transactions on Biomedical Engineering, vol. 64, pp. 28722879, 2017.

[23] N. K. Al-Qazzaz, M. K. Sabir, and K. Grammer, "Gender Differences identification from Brain Regions using Spectral Relative Powers of Emotional EEG," in Proceedings of the 2019 7th International work-conference on Bioinformatics and biomedical engineering, 2019, pp. 38-42.

[24] N. K. Al-Qazzaz, M. K. Sabir, and K. Grammer, "Correlation Indices of Electroencephalogram-Based Relative Powers during Human Emotion Processing," in Proceedings of the 2019 9th International Conference on Biomedical Engineering and Technology, 2019, pp. 64-70.

[25] N. K. Al-Qazzaz, M. K. Sabir, S. Ali, S. A. Ahmad, and K. Grammer, "Effective EEG Channels for Emotion Identification over the Brain Regions using Differential Evolution Algorithm," in 2019 41th Annual International Conference of the IEEE Engineering in Medicine and Biology Society (EMBC), 2019. 
[26] H. Azami and S. Sanei, "Spike detection approaches for noisy neuronal data: assessment and comparison," Neurocomputing, vol. 133, pp. 491-506, 2014.

[27] N. Kannathal, M. L. Choo, U. R. Acharya, and P. Sadasivan, "Entropies for detection of epilepsy in EEG," Computer methods and programs in biomedicine, vol. 80 , pp. 187194, 2005.

[28]J. Havrda and F. Charvát, "Quantification method of classification processes. Concept of structural \$ a \$-entropy," Kybernetika, vol. 3, pp. (30)-35, 1967.

[29] Z. Daróczy, "Generalized information functions," Information and control, vol. 16, pp. 36-51, 1970.
[30] S. Robert, "The Tsallis entropy of natural information [J]," Physica A: Statistical Mechanics and Its Applications, vol. 386, pp. 101-118, 2007.

[31] N. K. Al-Qazzaz, S. Ali, S. A. Ahmad, and J. Escudero, "Classification enhancement for post-stroke dementia using fuzzy neighborhood preserving analysis with QRdecomposition," in 2017 39th Annual International Conference of the IEEE Engineering in Medicine and Biology Society (EMBC), 2017, pp. 3174-3177.

[32] S. Dangel, P. Meier, H. Moser, S. Plibersek, and Y. Shen, "Time series analysis of sleep EEG," Computer assisted Physics, pp. 93-95, 1999. 


\title{
علامات هيرست و إنتروبيا تساليس لاكتشاف الصرع عند الطفال
}

\author{
سمية حمد جعفر

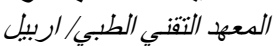

sumaya.hamad@epu.edu.iq:البريد الاكتروني

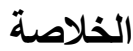

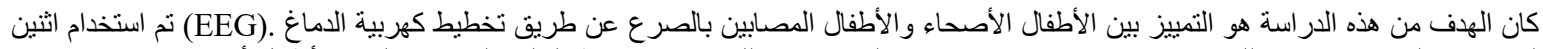

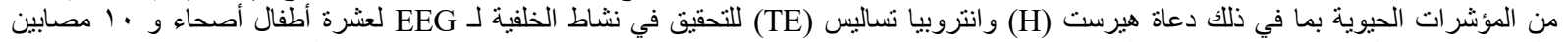

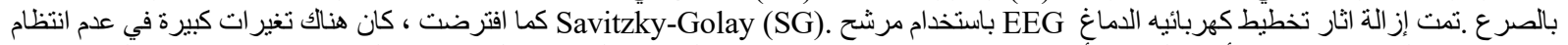

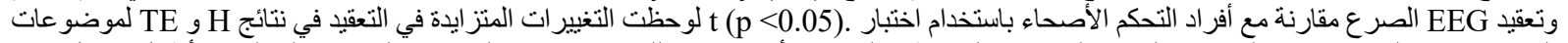

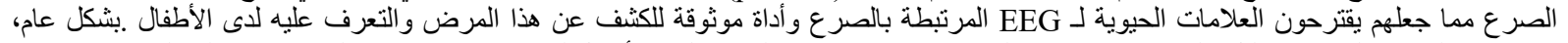

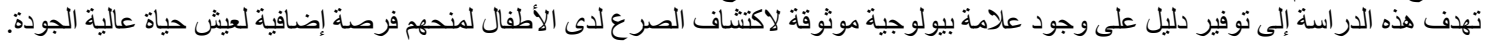

